\title{
An Algorithm for Extracting the Geometric Parameters of a Right Circular Cylinder from the Coefficients of its Algebraic Equation, and for a Cylinder/not a Cylinder Classification
}

\author{
Mohamed Ali Said \\ Eng. Math. \& Phys. Dept., Faculty \\ of Engineering, Zagazig University, \\ Egypt.

$$
\begin{aligned}
& \left(x-x_{0}\right)^{2}+\left(y-y_{0}\right)^{2}+\left(z-z_{0}\right)^{2}-R^{2}= \\
& \frac{\left[\lambda\left(x-x_{0}\right)+\mu\left(y-y_{0}\right)+v\left(z-z_{0}\right)\right]^{2}}{\lambda^{2}+\mu^{2}+v^{2}}
\end{aligned}
$$

\begin{abstract}
In this paper, an algorithm is introduced to serve in two tasks. The first is to extract the geometric parameters of a right circular cylinder from the coefficients of its algebraic equation. The second is to identify right circular cylinders from other quadrics given by an algebraic equation; that is, the algorithm can be used as a cylinder/not a cylinder classification tool. The algorithm is tested on a number of cases and its powerful is proved.
\end{abstract}

Keywords: Algorithm, Cylinder, Parameters, Classification.

\section{Introduction}

A right circular cylinder is one of the most important geometric primitives used in applications; a 95\% of industrial objects can be described by spheres, planes, cones, cylinders, and tori $[1,2]$.

Right circular cylinders are members of a larger family of surfaces, called quadrics. So, its algebraic equation takes the form [3]

$$
\begin{aligned}
& A x^{2}+B y^{2}+C z^{2}+D x y+E x z+F y z+G x+H y+ \\
& I z+J=0
\end{aligned}
$$

Where the coefficients $A, B, \ldots, J \in \mathbb{R}$, have little direct insight to the geometry of the surface. The conditions under which equation (1) represents a cylinder is given in [4], which is tedious. The proposed algorithm may be used to answer the classification question; whether a given quadric equation represents a right circular cylinder or not. The right circular cylinder is described by a set of parameters called the geometric parameters. These parameters are: a vector $\langle\lambda, \mu, v\rangle$ giving the direction of its axis, a point $\left(x_{0}, y_{0}, z_{0}\right)$ to fix the axis position, and a positive real number $R$ giving the radius of the cylinder. The geometric equation of the circular cylinder, in which the geometric parameters appear, is [5]:
The problems that will be addressed in this paper are how to find the geometric parameters of a circular cylinder from its algebraic equation, and how to identify a right circular cylinder from other quadrics given by an algebraic equation.

In literature, cylinders are subject of active research in many directions. Computing Cylinders from Minimal Sets of 3D Points [6, 7, 8]. Finding the Smallest Enclosing Cylinders to a set of data points $[9,10,11]$. Cylindrical objects detection, recognition and extraction $[2,12,13$, $14,15]$. Fitting of a cylinder to a set of data points [16, $17,18,19]$. In all this work, the geometric parameters of a cylinder play a central role.

It's hoped that the algorithm introduced in this paper enriches these efforts in two ways, first extracting the geometric parameters of a right circular cylinder from the coefficients of its algebraic equation, second identifying the right circular cylinder from other quadrics defined by the algebraic equation of quadrics.

The remaining of the paper is arranged as follows: section 2 is devoted to comparing coefficients of the algebraic and the geometric equations, section 3 is devoted for extracting the components of the axis direction-vector, section 4 is devoted for extracting the coordinates of a point on the cylinder axis, in section 5 the radius of the circular cylinder is extracted, in section 6 the proposed algorithm is introduced, section 7 is devoted for testing examples, and section 8 is devoted for conclusion. In the rest of this paper, cylinder means a right circular cylinder. 


\section{Comparing Coefficients of the Algebraic and the Geometric Equations}

Expanding equation (2), it takes the form

$$
\begin{aligned}
& \left(\mu^{2}+v^{2}\right) x^{2}+\left(\lambda^{2}+v^{2}\right) y^{2}+\left(\lambda^{2}+\mu^{2}\right) z^{2}-2(\lambda \mu) x y- \\
& 2(\lambda v) x z-2(\mu \nu) y z+2\left[\lambda\left(\mu y_{0}+v z_{0}\right)-\left(\mu^{2}+\right.\right. \\
& \left.\left.v^{2}\right) x_{0}\right] x+2\left[\mu\left(\lambda x_{0}+v z_{0}\right)-\left(\lambda^{2}+v^{2}\right) y_{0}\right] y+ \\
& 2\left[v\left(\lambda x_{0}+\mu y_{0}\right)-\left(\lambda^{2}+\mu^{2}\right) z_{0}\right] z+\left[\left(\mu^{2}+v^{2}\right) x_{0}^{2}+\right. \\
& \left(\lambda^{2}+v^{2}\right) y_{0}^{2}+\left(\lambda^{2}+\mu^{2}\right) z_{0}^{2}-2(\lambda \mu) x_{0} y_{0}-2(\lambda v) x_{0} z_{0}- \\
& \left.2(\mu v) y_{0} z_{0}-\left(\lambda^{2}+\mu^{2}+v^{2}\right) R^{2}\right]=0
\end{aligned}
$$

Comparing the coefficients of equation (1) and equation (3), then

$$
\begin{gathered}
A=\mu^{2}+v^{2}, \quad B=\lambda^{2}+v^{2}, \quad C=\lambda^{2}+\mu^{2} \\
D=-2 \lambda \mu, \quad E=-2 \lambda \nu, \quad F=-2 \mu \nu \\
G=-2\left(\mu^{2}+v^{2}\right) x_{0}+2 \lambda \mu y_{0}+2 \lambda v z_{0} \\
H=2 \mu \lambda x_{0}-2\left(\lambda^{2}+v^{2}\right) y_{0}+2 \mu v z_{0} \\
I=2 v \lambda x_{0}+2 v \mu y_{0}-2\left(\lambda^{2}+\mu^{2}\right) z_{0}
\end{gathered}
$$

$$
J=\left(\mu^{2}+v^{2}\right) x_{0}^{2}+\left(\lambda^{2}+v^{2}\right) y_{0}^{2}+\left(\lambda^{2}+\mu^{2}\right) z_{0}^{2}-
$$$$
2 \lambda \mu x_{0} y_{0}-2 \lambda v x_{0} z_{0}-2 \mu v y_{0} z_{0}-\left(\lambda^{2}+\mu^{2}+v^{2}\right) R^{2}
$$

\section{Extracting the Components of the Axis Direction- Vector}

In this section, the components $\lambda, \mu$, and $v$ will be extracted. Solving simultaneously equations (4), then

$$
\begin{gathered}
\lambda^{2}=\frac{-A+B+C}{2} \Rightarrow \lambda= \pm \sqrt{\frac{-A+B+C}{2}},(-A+B+C) \geq 0 \\
\mu^{2}=\frac{A-B+C}{2} \Rightarrow \mu= \pm \sqrt{\frac{A-B+C}{2}}, \quad(A-B+C) \geq 0 \\
v^{2}=\frac{A+B-C}{2} \Rightarrow v= \pm \sqrt{\frac{A+B-C}{2}},(A+B-C) \geq 0
\end{gathered}
$$

A use is made for equations (5) to fix the signs of $\lambda, \mu$, and $v$ since there are eight possibilities. Let the positive $\lambda$ be denoted by $\lambda_{+}$and the negative $\lambda$ by $\lambda_{-}$and similarly with $\mu$ and $v$, then use the following table to find the correct values.

Table 1: Determining $\lambda, \mu$, and $v$

\begin{tabular}{|c|c|c|c|c|c|c|c|c|}
\hline$\lambda$ & $\mu$ & $v$ & $\lambda \mu$ & $\lambda v$ & $\mu \nu$ & $-D / 2$ & $-E / 2$ & $-F / 2$ \\
\hline$\lambda_{+}$ & $\mu_{+}$ & $v_{+}$ & & & & & & \\
\hline$\lambda_{+}$ & $\mu_{+}$ & $v_{-}$ & & & & & & \\
\hline$\lambda_{+}$ & $\mu_{-}$ & $v_{+}$ & & & & & & \\
\hline$\lambda_{+}$ & $\mu_{-}$ & $v_{-}$ & & & & & & \\
\hline$\lambda_{-}$ & $\mu_{+}$ & $v_{+}$ & & & & & & \\
\hline$\lambda_{-}$ & $\mu_{+}$ & $v_{-}$ & & & & & & \\
\hline$\lambda_{-}$ & $\mu_{-}$ & $v_{+}$ & & & & & & \\
\hline$\lambda_{-}$ & $\mu_{-}$ & $v_{-}$ & & & & & & \\
\hline
\end{tabular}

The correct values are those in $\operatorname{row}(\mathrm{s})$ in which the middle three columns are matched with the last three columns. There will be two matches since a vector with opposite sense can also be used.

\section{Extracting the Coordinates of a Point on the Cylinder Axis}

In this section, the coordinates $x_{0}, y_{0}$, and $z_{0}$ of a point on the cylinder axis is determined. It is clear that there are infinity of points on the axis, any one of them can be used. Solving equations (6), (7), and (8) simultaneously for $x_{0}$, $y_{0}$, and $z_{0}$ using Gauss-Jordan elimination:

$$
\begin{aligned}
& {\left[\begin{array}{ccccc}
-\left(\mu^{2}+v^{2}\right) & \lambda \mu & \lambda \nu & \vdots & G / 2 \\
\lambda \mu & -\left(\lambda^{2}+v^{2}\right) & \mu \nu & \vdots & H / 2 \\
\lambda \nu & \mu \nu & -\left(\lambda^{2}+\mu^{2}\right) & \vdots & I / 2
\end{array}\right] \sim} \\
& {\left[\begin{array}{ccrcc}
1 & 0 & -\frac{\lambda}{v} & \vdots & \frac{\left(\lambda^{2}+v^{2}\right) G+\lambda \mu H}{-2 v^{2}\left(\lambda^{2}+\mu^{2}+v^{2}\right)} \\
0 & 1 & -\frac{\mu}{v} & \vdots & \frac{\lambda \mu G+H\left(\mu^{2}+v^{2}\right)}{-2 v^{2}\left(\lambda^{2}+\mu^{2}+v^{2}\right)} \\
0 & 0 & 0 & \vdots & \frac{\left(\mu^{2}+v^{2}\right)(\lambda G+\mu H+v I)}{2 v\left(\lambda^{2}+\mu^{2}+v^{2}\right)}
\end{array}\right]}
\end{aligned}
$$

where $v \neq 0$ and $\lambda^{2}+\mu^{2}+v^{2} \neq 0$. The system has no solution. The only way to force the system to have infinity of solutions, as expected, is to set

$$
\lambda G+\mu H+v I=0
$$

and thus the solutions are:

$$
\begin{gathered}
x_{0}=\frac{\lambda}{v} z_{0}-\frac{\lambda \mu H+\left(\lambda^{2}+v^{2}\right) G}{2 v^{2}\left(\lambda^{2}+\mu^{2}+v^{2}\right)}, y_{0}=\frac{\mu}{v} z_{0}-\frac{\lambda \mu G+H\left(\mu^{2}+v^{2}\right)}{2 v^{2}\left(\lambda^{2}+\mu^{2}+v^{2}\right)}, \\
z_{0}=z_{0}
\end{gathered}
$$

where $z_{0}$ is an arbitrary real number. Similarly, with different pivoting, two different sets of solutions are obtained: Under the conditions $\lambda \neq 0, \lambda^{2}+\mu^{2}+v^{2} \neq 0$, and $\lambda G+\mu H+v I=0$. The following set of solutions is obtained. 


$$
\begin{gathered}
x_{0}=x_{0}, y_{0}=\frac{\mu}{\lambda} x_{0}-\frac{\mu \nu I+\left(\lambda^{2}+\mu^{2}\right) H}{2 \lambda^{2}\left(\lambda^{2}+\mu^{2}+v^{2}\right)}, z_{0}=\frac{v}{\lambda} x_{0}- \\
\frac{\mu \nu H+\left(\lambda^{2}+v^{2}\right) I}{2 \lambda^{2}\left(\lambda^{2}+\mu^{2}+v^{2}\right)}
\end{gathered}
$$

where $x_{0}$ is an arbitrary real number and under the conditions $\mu \neq 0, \lambda^{2}+\mu^{2}+v^{2} \neq 0$, and

$$
\lambda G+\mu H+\nu I=0
$$

The following set of solutions is obtained

$$
\begin{gathered}
x_{0}=\frac{\lambda}{\mu} y_{0}-\frac{\lambda v I+\left(\lambda^{2}+\mu^{2}\right) G}{2 \mu^{2}\left(\lambda^{2}+\mu^{2}+v^{2}\right)}, y_{0}=y_{0}, \quad z_{0}=\frac{v}{\mu} y_{0}- \\
\frac{\lambda v G+\left(\mu^{2}+v^{2}\right) I}{2 \mu^{2}\left(\lambda^{2}+\mu^{2}+v^{2}\right)}
\end{gathered}
$$

where $y_{0}$ is an arbitrary real number.

The equations $(14-16)$ will be simplified by setting the arbitrary values to zeros, using $\lambda G+\mu H+v I=0$, and $2\left(\lambda^{2}+\mu^{2}+v^{2}\right)=A+B+C \neq 0:$

Case $1: \lambda \neq 0$

$x_{0}=0, \quad y_{0}=\frac{\mu G-\lambda H}{\lambda(A+B+C)}, \quad z_{0}=\frac{\nu G-\lambda I}{\lambda(A+B+C)}$

Case 2: $\mu \neq 0$

$x_{0}=\frac{\lambda H-\mu G}{\mu(A+B+C)}, \quad y_{0}=0, \quad z_{0}=\frac{v H-\mu I}{\mu(A+B+C)}$

Case 3: $v \neq 0$

$$
x_{0}=\frac{\lambda I-v G}{v(A+B+C)}, \quad y_{0}=\frac{\mu I-v H}{v(A+B+C)}, \quad z_{0}=0
$$

\section{Extracting the Radius of the Right Circular Cylinder}

The radius $R$ will be obtained using:

$R^{2}=\frac{2}{A+B+C}\left(A x_{0}^{2}+B y_{0}^{2}+C z_{0}^{2}+D x_{0} y_{0}+E x_{0} z_{0}+\right.$ $\left.F y_{0} z_{0}-J\right)$

\section{An Algorithm for Finding the Geometric Parameters of a Right Circular Cylinder, and for the Classification a Cylinder/not a Cylinder}

The condition of solvability stated in equation (13), $\lambda G+\mu H+\nu I=0$, can be expressed using the coefficients of the algebraic equations by utilizing equations $(4,5)$ as follows

$D E G+D F H+E F I=0$

\section{The Algorithm}

\section{START}

Step 1: Input the coefficients of the algebraic equation; $A, B, C, D, E, F, G, H, I, J$

Step 2: Determining $\lambda, \mu$, and $v$

$$
\begin{array}{ll}
\text { If }(-A+B+C)<0 \quad \text { OR } & (A-B+C)<0 \\
\text { OR }(A+B-C)<0 \text { then } & \text { output "Not a } \\
\text { Cylinder" and Stop } &
\end{array}
$$

Else

$$
\begin{aligned}
& \lambda_{+}=+\sqrt{\frac{-A+B+C}{2}}: \lambda_{-}=-\sqrt{\frac{-A+B+C}{2}}: \\
& \mu_{+}=+\sqrt{\frac{A-B+C}{2}} \\
& \mu_{-}=-\sqrt{\frac{A-B+C}{2}}: v_{+}=+\sqrt{\frac{A+B-C}{2}}: \\
& v_{-}=-\sqrt{\frac{A+B-C}{2}}
\end{aligned}
$$

End If

Fill in the following table

\begin{tabular}{|c|c|c|c|c|c|c|c|c|}
\hline$\lambda$ & $\mu$ & $v$ & $\lambda \mu$ & $\lambda v$ & $\mu \nu$ & $-D / 2$ & $-E / 2$ & $-F / 2$ \\
\hline$\lambda_{+}$ & $\mu_{+}$ & $v_{+}$ & & & & & & \\
\hline$\lambda_{+}$ & $\mu_{+}$ & $v_{-}$ & & & & & & \\
\hline$\lambda_{+}$ & $\mu_{-}$ & $v_{+}$ & & & & & & \\
\hline$\lambda_{+}$ & $\mu_{-}$ & $v_{-}$ & & & & & & \\
\hline$\lambda_{-}$ & $\mu_{+}$ & $v_{+}$ & & & & & & \\
\hline$\lambda_{-}$ & $\mu_{+}$ & $v_{-}$ & & & & & & \\
\hline$\lambda_{-}$ & $\mu_{-}$ & $v_{+}$ & & & & & & \\
\hline$\lambda_{-}$ & $\mu_{-}$ & $v_{-}$ & & & & & & \\
\hline
\end{tabular}

Find the matches between the middle three columns and the last three columns then $\lambda=\cdots: \mu=\cdots: v=\cdots$

If there is no matching then output "Not a cylinder" and stop

Step 3: Determining $x_{0}, y_{0}$, and $z_{0}$

If $A+B+C \neq 0$ and $\lambda G+\mu H+\nu I=0$ then If $\lambda \neq 0$ then

$$
x_{0}=0 \quad: \quad y_{0}=\frac{\mu G-\lambda H}{\lambda(A+B+C)} \quad: \quad z_{0}=\frac{v G-\lambda I}{\lambda(A+B+C)}
$$

Else if $\mu \neq 0$ then

$$
x_{0}=\frac{\lambda H-\mu G}{\mu(A+B+C)} \quad: \quad y_{0}=0 \quad: \quad z_{0}=\frac{v H-\mu I}{\mu(A+B+C)}
$$

Else If $v \neq 0$ then

$$
x_{0}=\frac{\lambda I-v G}{v(A+B+C)} \quad: \quad y_{0}=\frac{\mu I-v H}{v(A+B+C)} \quad: \quad z_{0}=0
$$

Else

Output " Not a cylinder" and stop

End if

Else

Output "Not a cylinder" and stop

End if 
Step 4: Determining $R$

If

$\frac{2}{A+B+C}\left(\begin{array}{c}A x_{0}^{2}+B y_{0}^{2}+C z_{0}^{2}+D x_{0} y_{0}+E x_{0} z_{0} \\ +F y_{0} z_{0}-J\end{array}\right) \geq 0$ then

$R=$

$\sqrt{\frac{2}{A+B+C}\left(A x_{0}^{2}+B y_{0}^{2}+C z_{0}^{2}+D x_{0} y_{0}+E x_{0} z_{0}+F y_{0} z_{0}-J\right)}$

Else

Output "Not a Cylinder" and stop

End if

Step 5: Output $\lambda, \mu, v, x_{0}, y_{0}, z_{0}, R$

\section{END}

\section{Testing Examples}

\subsection{A simple case}

$x^{2}+y^{2}-4=0$.

Applying the algorithm, the results are:

$\lambda=0, \quad \mu=0, \quad v=1, \quad x_{0}=0, \quad y_{0}=0, \quad z_{0}=0$,

$R=2$.

\subsection{A medium case}

$$
13 x^{2}+10 y^{2}+5 z^{2}-4 x y-6 x z-12 y z-56=0
$$

Applying the algorithm, the results are:

$\lambda=1, \quad \mu=2, \quad v=3, \quad x_{0}=0, \quad y_{0}=0, \quad z_{0}=0$, $R=2$.

\subsection{A hard case $[20]$}

$$
\begin{aligned}
392 x^{2}+596 y^{2}+ & 596 z^{2}-560 x y-560 x z-392 y z \\
& +6048 x-5112 y-3528 z+15127 \\
& =0
\end{aligned}
$$

Applying the algorithm, the results are:

$$
\begin{array}{ll}
\lambda=20, \quad \mu=14, \quad v=14, \quad x_{0}=0, \quad y_{0}=5.9, \\
z_{0}=4.9, \quad R \approx 3.295 .
\end{array}
$$

\subsection{Not a cylinder case}

A unit sphere: $x^{2}+y^{2}+z^{2}-1=0$.

Applying the algorithm, the results are:

$\lambda=\mu=v= \pm 1 / \sqrt{2} \Rightarrow \lambda \mu=\lambda \nu=\mu \nu \neq 0$, whereas

$-\frac{D}{2}=-\frac{E}{2}=-\frac{F}{2}=0$

There is no matching $\Rightarrow$ not a cylinder.

\subsection{Another not a cylinder case}

A cone: $x^{2}+y^{2}-z^{2}=0$.

Applying the algorithm, the results are:

$\lambda$ is imaginary $\Rightarrow$ not a cylinder.

\section{Conclusion}

A right circular cylinder is one of the most important geometric primitives used in applications. In literature, cylinders are a subject of active research in many directions. Computing cylinders from minimal sets of 3D points. Finding the smallest enclosing cylinders to a set of data points. Cylindrical objects detection, recognition and extraction. Fitting a cylinder to a set of data points. In all this work, the geometric parameters of a cylinder play a central role. The right circular cylinder is described by a set of parameters called the geometric parameters. These parameters are: a vector $\langle\lambda, \mu, v\rangle$ giving the direction of its axis, a point $\left(x_{0}, y_{0}, z_{0}\right)$ to fix the axis position, and a positive real number $R$ giving the radius of the cylinder. The algebraic equation of a right circular cylinder, as a member of quadric surfaces, is given and its geometric equation. The coefficients of the two equations are compared resulting-in ten equations. The geometric parameters are expressed in terms of the coefficients of the algebraic equations in three stages. In the first stage the components of the axis-direction vector is obtained using a table to account for the signs of the components in such a way that the results satisfy six equations. In the second stage the coordinates of a point on the axis is obtained through solving three equations that are incompatible and to solve the incompatibility, three conditions are set on the coefficients of the three equations, then three sets of solutions are obtained and only one of them is used according to the case considered. In the third stage the radius of the cylinder is obtained.

Finally, the proposed algorithm is introduced. The algorithm serves in two ways. The first way is in extracting the geometric parameters of a right circular cylinder from the coefficients of its algebraic equation. The second way is in identifying the right circular cylinder from other quadrics defined by an algebraic equation; that is, as a classification tool.

\section{References}

[1] Z. Toony et al., "PGP2X: Principal Geometric Primitives Parameters Extraction", In: Proceedings of the $10^{\text {th }}$ International Joint Conference on Computer Vision, Imaging and Computer Graphics Theory and Applications (VISIGRAPP2015), Berlin, Germany, March 2015.

[2] T. Tran et al., "Extraction of cylinders and estimation of their parameters from point clouds", Computers \& Graphics, Vol. 46, pp. 345-357, February 2015.

[3] V. A. Ilyin and E. G. Poznyak, “Analytic Geometry”, pp. 191, Mir Publishers, Moscow 1984.

[4] M. E. Mortenson, "Computer Graphics Handbook: Geometry and Mathematics”, pp. 167, Industrial Press Inc., New York 1990.

[5] A. V. Pogorelov, “Analytic Geometry”, pp. 154, Mir Publishers, Moscow 1980.

[6] C. Beder and W. Forstner, "Direct Solutions for Computing Cylinders from Minimal Sets of 3D Points", In: Proceedings of European Conference on Computer Vision (ECCV2006), pp. 135-146, 2006.

[7] O. Devillers et al., "On Circular Cylinders By Four Or Five Points In Space”, Report No 4195, Institut National De Recherche En Informatique Et En Automatique, France, June 2001.

[8] D. Lichtblau, "Cylinders Through Five Points: Computational Algebra and Geometry", Journal of Mathematics Research, Vol. 4, No. 6, December 2012. 
[9] G. A. Watson, "Fitting enclosing cylinders to data in $R^{n}$ ", Numerical Algorithms, Vol. 43, No. 2, pp. 189-196, December 2006.

[10] E. Schomer et al., "Smallest Enclosing Cylinders", In: Proceedings of the twelfth annual symposium on computational geometry, January 1996.

[11] R. Brandenberg and T. Theobald, "Algebraic Methods for Computing Smallest Enclosing and Circumscribing Cylinders of Simplices", Applicable Algebra in Engineering, Communication and Computing (AAECC), Vol. 14, No. 6, pp. 439-460, March 2004.

[12] R. Figueiredo et al., "Robust cylinder detection and pose estimation using $3 D$ point cloud information", In: Proceedings of IEEE International Conference on Autonomous Robot Systems and Competitions (ICARSC),pp. 234-239, April 26-28, Coimbra, Portugal, 2017.

[13] M.M.M.Sarcar et al., " Automatic Feature Recognition of Cylinder and Knuckle Thread from Neutral Files", 5th International \& 26th All India Manufacturing Technology, In: Proceedings of Design and Research Conference (AIMTDR 2014), December 12th-14th, IIT Guwahati, Assam, India, 2014.

[14] T. Chaperon and F. Goulette, "Extracting cylinders in full $3 D$ data using a random sampling method and the Gaussian image", In: Proceedings of Vision Modeling and Visualization Conference 2001 (VMV-01), pp. 35-42, Stuttgart, Germany, Nov 2001

[15] T. Rabbani and F. Heuvel, "Efficient Hough Transform for Automatic Detection of Cylinders in Point Clouds", In: Proceedings of ISPRS WG III/3, III/4, V/3 Workshop "Laser scanning 2005", Enschede, pp. 60-65, the Netherlands, September 12-14, 2005.

[16] G. Lukacs et al., "Faithful Least-Squares Fitting of Spheres, Cylinders", Cones and Tori for Reliable Segmentation, In: Proceedings of $5^{\text {th }}$ European Conference on Computer Vision (ECCV'98), pp. 671-686, Freiburg, Germany, June 2-6, 1998.

[17] I. A. Al-Subaihi, "Geometric Fitting by two Coaxial Cylinders", Mathematical Communications, Vol. 21, pp. 227237, 2016.

[18] A. Nurunnabi et al., "Robust cylinder fitting in threedimensional point cloud data", International Archives of the Photogrammetry, Remote Sensing and Spatial Information Sciences, Volume XLII-1/W1, pp. 63-70, 2017.

[19] S. Kwon et al., " Algorithms for fitting cylindrical objects to sparse range point clouds for rapid workspace modeling", In: Proceedings of $20^{\text {th }}$ International Symposium on Automation and Robotics in Construction, pp. 173-178, September 2003.

[20] M. Afwat, "Geometry part II", pp. 143, Zagazig University, Egypt, 1978. 